\title{
Validation of an HPLC-DAD/UV method for the quantification of cyclamate in tabletop sweeteners: risk of exceeding the acceptable daily intake
}

\section{B. Sargaço, C. Serra \& E. Vasco}

To cite this article: B. Sargaço, C. Serra \& E. Vasco (2017) Validation of an HPLC-DAD/ UV method for the quantification of cyclamate in tabletop sweeteners: risk of exceeding the acceptable daily intake, Food Additives \& Contaminants: Part A, 34:6, 883-890, DOI: 10.1080/19440049.2017.1306756

To link to this article: http://dx.doi.org/10.1080/19440049.2017.1306756

Published online: 11 Apr 2017.

Submit your article to this journal $\sqsubset$

Џll Article views: 82

View related articles $[3$

View Crossmark data ¿ 


\title{
Validation of an HPLC-DAD/UV method for the quantification of cyclamate in tabletop sweeteners: risk of exceeding the acceptable daily intake
}

\author{
B. Sargaço ${ }^{a}$ C. Serra ${ }^{a}$ and E. Vasco ${ }^{b}$
}

${ }^{a}$ Chemical Engineering and Biotechnology Research Center, Chemical Engineering Department, High Institute of Engineering of Lisbon, Lisbon, Portugal; b Food and Nutrition Department, National Health Institute Dr. Ricardo Jorge, Lisbon, Portugal

\begin{abstract}
Cyclamate is widely used as intense sweetener in the European Union. The absence of a maximum limit for the use of cyclamate in tabletop sweeteners and the growing demand for this type of product highlights the importance of developing robust analytical methods for the determination of its content to understand if the consumption of tabletop sweeteners can have a negative impact on human health. The present work aimed at the optimisation and validation of an high-performance liquid chromatography (HPLC) analytical method for cyclamate determination in tabletop sweeteners based on the procedure of European Standard EN 12857. The validated method was then applied to the determination of this sweetener in different types of tabletop sweeteners (liquid, powder and tablets). Both standards and samples solutions were submitted to a derivatisation procedure which converted cyclamate to N,N-dichlorocyclohexylamine. The derivatised product was separated and quantified using a reversed-phase column, a mobile phase composed of water (20\%) and methanol (80\%), isocratic flow of $1 \mathrm{ml} \mathrm{min}^{-1}$, and detection by ultraviolet spectrophotometry at a wavelength of $314 \mathrm{~nm}$. The analytical method was internally validated according to the following validation parameters: working range, linearity, limits of detection and quantification, sensitivity, precision (repeatability and intermediate precision), and uncertainty. This method proved to be specific and selective for the determination of this sweetener, showing repeatability, $\mathrm{RSD}_{\mathrm{r}} \leq 3 \%$, intermediate precision, $\mathrm{RSD}_{\mathrm{R}} \leq 3.3 \%$, and recovery rates from $92 \%$ to $108 \%$ for the different tabletop sweeteners. The method uncertainty was $9.4 \%$. The concentration of cyclamate in the samples varied significantly, from $2.9 \%$ to $73.9 \%$, which demonstrated that a possible excessive consumption of one of the analysed sweeteners can lead to exceeding the acceptable daily intake for cyclamate.
\end{abstract}

\section{ARTICLE HISTORY}

Received 9 December 2016

Accepted 4 March 2017

\section{KEYWORDS}

Cyclamate; HPLC; validation; tabletop sweeteners; ADI

\section{Introduction}

Nowadays there is a wide range of different food products containing non-caloric high-intense sweeteners that constitute an attractive alternative to sugar consumption. Aromatic water-based beverages and tabletop sweeteners are the main applications (Zygler et al. 2011).

Although sometimes considered as modern ingredients, high-intense sweeteners have been used for over a century (EUFIC 2012). They can be produced synthetically from chemical compounds (e.g., cyclamate) or from natural substances (e.g., steviol glycosides). Because their sweetening power is several times higher than sucrose, they can be used in very low concentrations in food and beverages allowing a significant cut in production costs and a virtually zero calorie contribution to the final food product.
Thus, even if they have some caloric content, it becomes irrelevant to the final product because of the low amounts added (Szmrecsányi \& Alvarez 1998; ISO 2012).

Cyclamate was discovered in 1937; its approval and commercialisation in the United States, and later in the European Union, boosted the segment of 'light' products, making it attractive not only to the normal consumer but also for people with particular conditions such as diabetes (De La Peña 2010).

Despite the fact that its use has been banned in the United States since 1969 due to a controversial study that associated this sweetener with the development of bladder tumour in rats, it is the highintense sweetener with the largest production volume worldwide (Kishi et al. 2010).

Cyclamate has been approved in the European Union for general use since 1994 in accordance 
with Directives 94/35/EC (EC 1994) and 96/83/EC (EC 1996) with its last amendment by Regulations $1333 / 2008$ (EC 2008) and 1129/2011 (EC 2011). This legislation stipulates in which foodstuffs and beverages cyclamate can be used, the maximum amounts of use, and provides the purity criteria that must be met by producers, as well as additional information that must be included in the labelling (EC 1994, 1996, 2003).

Of all food categories approved for the use of cyclamate and other intense sweeteners, tabletop sweeteners is the only one for which there is no maximum permitted level (MPL) of use, nor a requirement for labelling its exact content in the respective package (EC 1994, 2008). Therefore, food producers may use the quantities they find necessary and advantageous to formulate a particular tabletop sweetener. This can result in undeniable risks to human health since the acceptable daily intake of $7 \mathrm{mg} \mathrm{kg}^{-1}$ body weight for cyclamate (SCF 2000) may run the risk of being surpassed in the case of excessive consumption.

The widespread use and consumption of cyclamate salts and the alleged adverse health effects compel the development and validation of efficient analytical methods for its precise determination (Huang et al. 2006).

From all the analytical methods developed over decades for the analysis of this sweetener in different food matrices, chromatographic methods have been the most popular, particularly HPLC (Kokotou 2013).

Several analytical methods based on HPLC with different detectors and pretreatment of samples have been developed for the determination of cyclamate, either individually or together with other sweeteners (Shah et al. 2014, 2015; Sakai et al. 2015). Detection by indirect spectrophotometry (Herrmann et al. 1983), absorption in the visible range with the addition of dyes (Choi et al. 2000), absorption in the ultraviolet range (UV) and fluorescence prior to sample derivatisation (Hauck \& Köbler 1990; Choi et al. 2000), MS (Zygler et al. 2011), a light-scattering evaporative detector (Wasik et al. 2007), and capillary zone electrophoresis (Vistuba et al. 2015) have been used.

In the European Union there are two official analytical procedures based on HPLC technology with UV detection published by the European Committee for Standardisation (CEN): EN 12857, which requires a pretreatment (derivatisation) of the samples, and EN 1379, which is specific for the analysis of liquid tabletop sweeteners preparations (EN 1379 1996, EN 12857 1999). EN 12857 uses a reaction with active chlorine to convert cyclamate to N,N-dichlorocyclohexylamine, which absorbs UV (EN 12857 1999).

The HPLC-UV method applied in the present work for the analysis of cyclamate was based on EN 12857. The method is very specific for this sweetener, can be applied in very different food matrices (EN 12857 1999), is less expensive and does not need a very specialised technician, as would be necessary with an HPLC-MS method.

In this way, the aim of this work was to optimise and validate an official method of HPLC-UV that could be applied not only in the analysis of tabletop sweeteners but also in other foodstuffs in order to provide an efficient and highly specific method for the food quality-control laboratory. Furthermore, with this work it will be possible to understand how cyclamate levels vary in different tabletop sweeteners and evaluate if the compliance of ADI value is at risk. The results may also allow future dietary intake estimation studies to be carried out in Portugal as they are non-existent with regard to the consumption of cyclamate and tabletop sweeteners.

\section{Materials and methods}

\section{Samples}

A total of 28 samples of one powder, one tablet and two liquid tabletop sweeteners brands were analysed. For each brand, five packs of one batch and a pack for each of two other batches, which amounted to seven packs for each sweetener brand, were purchased. All the tabletop sweeteners had cyclamate and were acquired in Lisbon supermarkets.

\section{Reagents and standard solutions}

Methanol (> 99\%), $n$-heptane (> 99\%) and 2-propanol (> 99.8\%), from Merck (Germany) and sodium carbonate (> 99.8\%) from, were of HPLC grade. All other reagents were of recognised analytical grade unless stated otherwise. Sodium sulphate (> 99\%) and sulphuric acid (96\%) was purchased from Merck (Germany) and sodium carbonate (> 99.8\%) from Riedel-de-Haën (Germany). Ultra-pure type I water, 
generated by a Milli-Q system, was used. The calcium cyclamate standard (> 99\%) was purchased from Sigma-Aldrich (USA). Sodium carbonate (5\%), sulphuric acid (50\%) solutions and cyclamic acid standard stock solution ( $2 \mathrm{mg} \mathrm{l}^{-1}$ ) were prepared by diluting with water. The standard solutions for the calibration curve were prepared by diluting the derivatised stock solution $\left(2 \mathrm{mg} \mathrm{l}^{-1}\right)$ into six volumetric flasks with $n$-heptane corresponding to the six calibration points of $40,70,100,130,160$ and $200 \mathrm{mg} \mathrm{l}^{-1}$ of cyclamic acid. Commercial bleach with active chloride was used for the derivatisation procedure.

\section{Sample treatment}

Liquids and powder tabletop sweeteners were homogenised manually. The tablet sweetener was milled and homogenised through the use of an automatic laboratory mill. No sample pretreatment was necessary and the homogenised samples were directly weighted $(0.5-1 \mathrm{~g})$ or pipetted $(0.5-1 \mathrm{ml})$ into volumetric flasks and diluted with water. Each sample was analysed in triplicate, two of them (duplicates) to account for any error occurring throughout the analytical procedure; the third was fortified. The purpose of the latter was to calculate the recovery rate of cyclamate.

\section{Derivatisation}

Both standard and sample solutions were submitted to a derivatisation procedure that converted cyclamate into N,N-dichlorocyclohexylamine. Sample or standard solution $(20 \mathrm{ml})$ were added to separating funnels, followed by $1 \mathrm{ml}$ of sulphuric acid (50\%), $10 \mathrm{ml}$ of $n$-heptane and $2.5 \mathrm{ml}$ of bleach. After shaking and separation of the organic from the aqueous phase, $25 \mathrm{ml}$ of sodium carbonate solution $\left(50 \mathrm{~g} \mathrm{l}^{-1}\right)$ were added. Again, the funnels were shaken, the lower phase discarded and the $n$-heptane phase dried with approximately $1 \mathrm{~g}$ of sulphate sodium. The solution was filtered by fluted filter paper and through a $0.45-\mu \mathrm{m}$ membrane filter before loading the vials onto the HPLC system.

\section{HPLC analysis}

For the chromatographic analysis a LiChrospher 60 RP-select B $(5 \mu \mathrm{m}$ particle size) $250 \times 4 \mathrm{~mm}$ column at $37^{\circ} \mathrm{C}$ was used in a Waters A2690 HPLC system equipped with a sample cooler, a column heater/ cooler and a 2996 photodiode array detector. Detection was performed at a wavelength of $314 \mathrm{~nm}$. The mobile phase was an isocratic mixture of methanol and water (80:20) with a flow rate of

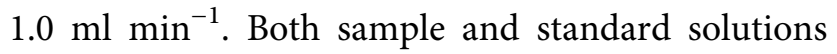
were injected in duplicate with a volume of $20 \mu \mathrm{l}$ and a total run time of 8 min was applied.

Data were collected and analysed using Water's Empower 2 chromatography data software. The derivatised product was identified by comparing sample and standard retention times and the quantification was made by external standard calibration using sample peak areas which were interpolated in the equation of the calibration curve. The cyclamic acid concentration was expressed in $\mathrm{mg} \mathrm{kg}^{-1}$.

\section{Method optimisation, validation and analytical quality assurance}

Before proceeding to an in-house validation, some analytical tests and critical analyses were carried out, focusing on the procedure and chromatographic conditions recommended by EN 12857. In order to ensure the quality of the method's measurements, an in-house validation was carried out, in line with the following parameters: calibration curve (working range, linearity and sensitivity), LOD, LOQ, repeatability and intermediate precision, recovery, accuracy and uncertainty. The working range was established according to the homogeneity of variances test using an $F$-test. Linearity was evaluated by taking into account the determination coefficient of the calibration curves using statistical tests such as residual analysis and Mandel's test. The method's sensitivity was accessed by checking the slope's variation in several calibration curves. The analytical limits were determined using the slope $(a)$ of the calibration curve and its residual standard deviation $\left(S_{y / x}\right)$, which were later experimentally verified. The method's repeatability and intermediate precision were expressed as a relative standard deviation, $\mathrm{RSD}_{\mathrm{r}}$ and $\mathrm{RSD}_{\mathrm{R}}$, respectively, for the three sample matrices in the study. Due to the lack of certified reference materials containing cyclamate, the method's accuracy was checked through participation in Food Analysis Performance Assessment Scheme (FAPAS) proficiency tests which provide good 
evidence of internal and external quality control assessment. Uncertainty of the measurements was quantified using method validation data, namely intermediate precision and accuracy. The combined standard uncertainty was then calculated and expanded using a coverage factor, $k=2$ (IPAC 2007).

\section{Sample homogeneity}

Cyclamate homogeneity was assessed by the analysis of variance (ANOVA) test, which was used to compare means and variations between samples of the same batch and different batches of the four tabletop sweetener brands in study. This demonstrated whether cyclamate concentration levels are statistically equal, that is, if they do not differ significantly in the same brand.

\section{Stability of the derivatised solutions}

According to the EN 12857, the derivatised solutions are only stable for approximately $24 \mathrm{~h}$ when stored at $4^{\circ} \mathrm{C}$. This represents an important limitation to the method's practicability since it requires that new calibration solutions must be prepared at each new analytical assay to carry out good analytical results. In this way, the stability of the derivatised solutions was evaluated by preparing the usual six-point calibration curve and injecting them on the same day. More replicates of these calibration solutions were stored in different conditions and during different time periods and analysed afterwards. In this stability study the chromatographic peak areas of the replicates were compared with those obtained for the first set of calibration solutions.

\section{Results and discussion}

\section{Method optimisation}

Initially, the Norm indications were followed, which advises the preparation of the calibration solutions by different dilutions of the standard stock solution and subsequent derivatisation of each individual solution. The great variability associated with each individual assay enables the validation of a working range. The method optimisation allowed a new procedure regarding the preparation of the calibration solutions. A direct derivatisation of $20 \mathrm{ml}$ of the standard stock solution and subsequent dilutions with $n$-heptane were performed to obtain the established six calibration points. In this way, the new procedure includes only one derivatisation reaction to prepare each set of calibration curve solutions.

\section{Method validation and analytical quality assurance}

The working range was established by testing concentrations in the range between 20 and $800 \mathrm{mg} \mathrm{l}^{-1}$, and the interval of $40-200 \mathrm{mg} \mathrm{l}^{-1}$ for the calibration curve was chosen. Ideally a larger range would suit better the analysis of cyclamate in tabletop sweeteners because of its very high concentration, but according to the homogeneity test of variances it was not possible to validate a larger range. In this working range six concentration points were defined for the calibration line: 40,70,100, 130, 160 and $200 \mathrm{mg} \mathrm{l}^{-1}$.

The results of the applied statistical tests are presented in Table 1. The results from Mandel's test allowed us to conclude that the linear regression model is adequate. Furthermore as the residuals were always less than $10 \%$ and the $r^{2}$ was higher than 0.995; a good linear correlation between the chromatographic peaks areas and the concentration points was also proved.

Calibration curve slope variation showed that the sensitivity of the method remained constant throughout all assays.

The LOQ and LOD calculated using the calibration curve slope and residual standard deviation $\left(S_{y / x}\right)$ were always lower than the first calibration point (40 $\mathrm{mg} \mathrm{l}^{-1}$ ), which confirmed their adequacy.

Table 1. Validation parameters.

\begin{tabular}{|c|c|}
\hline \multicolumn{2}{|c|}{ Validation parameters } \\
\hline Calibration curve & $n=6$ \\
\hline Working range $\left(\mathrm{mg} \mathrm{I}^{-1}\right)$ & $40-200$ \\
\hline Homogeneity of variances & PG $(1.51)<F(5.35)$ \\
\hline Linearity (Mandel test) & $P G(0.11)<F(10.56)$ \\
\hline Residuals & $\leq 10 \%$ \\
\hline Determination coefficient $\left(r^{2}\right)$ & $\geq 0.995$ \\
\hline \multicolumn{2}{|l|}{ Analytical and method limits } \\
\hline Quantification & $40 \mathrm{mg} \mathrm{l}^{-1}$ and $4 \mathrm{~g} \mathrm{I}^{-1}$ \\
\hline Detection & $13.3 \mathrm{mg} \mathrm{l}^{-1}$ and $1.3 \mathrm{~g} \mathrm{l}^{-1}$ \\
\hline \multicolumn{2}{|l|}{ Sensitivity } \\
\hline Calibration curve's slope variation (CV) & $3.3 \%$ \\
\hline \multicolumn{2}{|l|}{ Selectivity } \\
\hline Peak visualisation & $\begin{array}{l}\text { No peak at retention time } \\
\text { of the analyte }\end{array}$ \\
\hline \multicolumn{2}{|l|}{ Accuracy } \\
\hline FAPAS proficiency test (Z-score) & $0.8 \leq 2$ \\
\hline
\end{tabular}


However, for practical reasons, it was decided to establish the first calibration point of $40 \mathrm{mg} \mathrm{l}^{-1}$ of cyclamic acid, and one-third of this value, as the analytical LOQ and LOD, respectively. To confirm the precision and accuracy of these limits, two tabletop sweetener sample solutions with no cyclamate in their content but fortified with the LOQ and LOD concentration were analysed. The recovery rate of the LOQ solution and the peak visualisation of the LOD allowed for the conclusion that these values were well established. Using these analytical limits and taking into account the dilution factors and the biggest amount of each sample weighted, the calculated method LOQ and LOD were 4 and $1.3 \mathrm{~g} \mathrm{l}^{-1}$, respectively.

The selectivity of the method was confirmed using the chromatograms obtained for the solution of a tabletop sweetener without cyclamate; no signal was observed at the retention time of $\mathrm{N}, \mathrm{N}-$ dichlorocyclohexylamine.

Regarding the method's precision, the $\mathrm{RSD}_{\mathrm{r}}$ were $3.0 \%, 3.0 \%$ and $1.3 \%$ for the powder, tablet and liquid 1 samples, and $\mathrm{RSD}_{\mathrm{R}}$ were $2.7 \%, 2.6 \%$ and $3.3 \%$ for the same tabletop sweeteners respectively (Table 2). These values are relatively low, which means that the cyclamate concentration determined in different types of sample in the same day under the same analytical conditions (repeatability) and on different days (intermediate precision) were very similar. Thus, the method showed good precision for the analysis of cyclamate in different tabletop sweeteners.

In the FAPAS proficiency test, one sample of soft drink containing cyclamate was analysed using the same analytical procedure. The $Z$-score of 0.8 attested good accuracy and showed that the method can be applied for the analysis of different food samples and matrices such as tabletop sweeteners and soft drinks.

The uncertainty of the measurements was determined from the intermediate precision data and using the uncertainty related to the results of the

Table 2. Precision values for different samples analysed.

\begin{tabular}{lcc}
\hline $\begin{array}{l}\text { Tabletop } \\
\text { sweetener }\end{array}$ & $\begin{array}{c}\text { Repeatability precision } \\
\mathrm{RSD}_{\mathrm{r}}(\%)\end{array}$ & $\begin{array}{c}\text { Intermediate precision } \\
\mathrm{RSD}_{\mathrm{R}}(\%)\end{array}$ \\
\hline Powder & 3.0 & 2.7 \\
Tablet & 3.0 & 2.6 \\
Liquid 1 & 1.3 & 3.3 \\
\hline
\end{tabular}

FAPAS proficiency test. The combined standard uncertainty was then calculated and expanded using a coverage factor, $k=2$, that provides $95 \%$ confidence. The quantification of uncertainty by this model showed that the two components, intermediate precision and accuracy, had similar contributions to combined standard uncertainty. In this way, the expanded measurement uncertainty was $9.4 \%$.

\section{Stability of the derivatised solutions}

A brief study regarding the stability of the derivatised solutions was carried out as previously explained. After comparing the chromatographic peak areas of the replicates with the those obtained for recently prepared calibration solutions, it was possible to state that the stability of the derivatised solutions is superior to that indicated in the European Norm. In fact, a maximum per cent deviation of about $3.3 \%$ was observed when storing the solutions at $4^{\circ} \mathrm{C}$ or at RT for $48 \mathrm{~h}$.

\section{Analysis of samples}

Analysis of tabletop sweetener samples was performed according to the validated method. Figure 1 shows one of the obtained sample chromatograms $\left(71 \mathrm{mg} \mathrm{l}^{-1}\right)$. Table 3 presents the cyclamic acid concentration in the different 28 packages of the four tabletop sweeteners and the corresponding recovery rates. Using the conversion factor of 0.8906 , which accounts for the difference in molecular mass between cyclamic acid and its sodium salt, it was possible to determine the sodium cyclamate content of each package. As criteria for acceptance of the results, for each duplicate analysis a maximum repeatability coefficient of variation of 5\% was established according to ISO 5725-6: 1994.

As shown in Table 3, the concentration of sodium cyclamate in the different tabletop sweeteners varies greatly, from $2.9 \%$ to $73.9 \%$, which was expected due to the absence of an MPL for cyclamate in this type of products. Also, compared with other intense sweeteners, cyclamate is usually used in much higher amounts due to its lower sweetness, which could explain the amounts found.

The recovery rates ranged between values close to $100 \%(92-108 \%)$, which allowed for the conclusion 


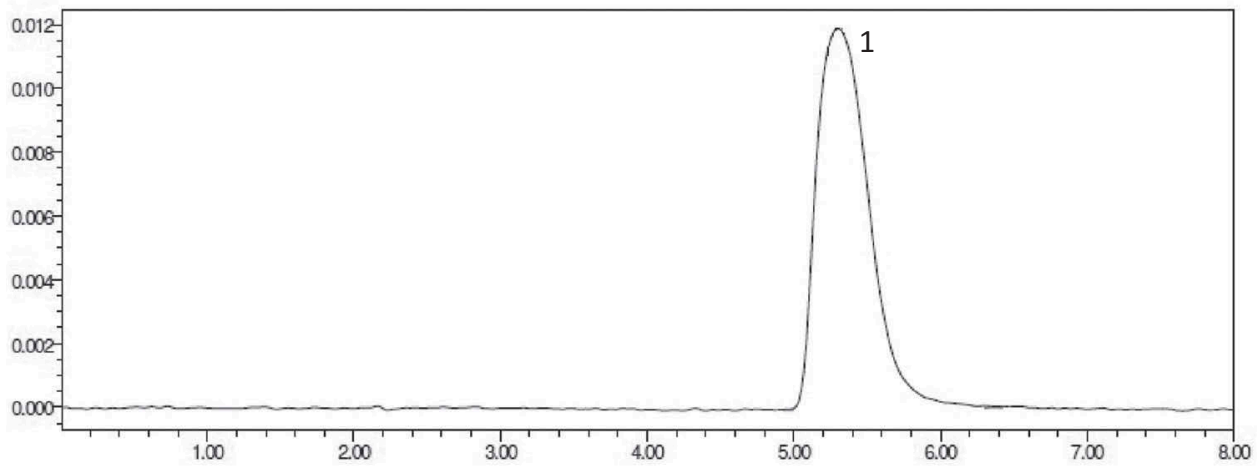

Figure 1. Powder sample chromatogram. $1=\mathrm{N}, \mathrm{N}$-dichlorocyclohexylamine; $\mathrm{RT}=5.3 \mathrm{~min}$.

Table 3. Cyclamic acid and sodium cyclamate concentration, recovery rates and label contents of the tabletop sweeteners analysed.

\begin{tabular}{|c|c|c|c|c|c|}
\hline \multirow[b]{2}{*}{ Tabletop sweetener } & \multirow{2}{*}{$\frac{\text { Cyclamic acid concentration range }}{\mathrm{g} \mathrm{kg}^{-1}}$} & \multirow{2}{*}{$\frac{\text { Recovery rate }}{\%}$} & \multicolumn{2}{|c|}{ Sodium cyclamate concentration range } & \multirow{2}{*}{$\frac{\text { Labelled content }}{\%}$} \\
\hline & & & $\mathrm{g} \mathrm{kg}^{-1}$ & $\%$ & \\
\hline Powder & $68-71$ & $93-102$ & $77-80$ & $7.7-8.0$ & 8 \\
\hline \multirow[t]{2}{*}{ Tablet } & $625-658$ & $96-107$ & $702-739$ & $70.2-73.9$ & Not specified \\
\hline & $\mathrm{g} \mathrm{I}^{-1}$ & & $\mathrm{~g} \mathrm{I}^{-1}$ & & \\
\hline Liquid 1 & $26-29$ & $98-108$ & $29-32$ & $2.9-3.2$ & Not specified \\
\hline Liquid 2 & $124-132$ & $92-106$ & $139-148$ & $13.9-14.8$ & 15 \\
\hline
\end{tabular}

that there were minimal analyte losses during each analytical procedure.

For the two tabletop sweeteners, powder and liquid 2 samples, for the labels that specified the exact amount of cyclamate there was a maximum deviation of only $0.3 \%$ and $1.1 \%$, respectively.

By comparing the concentration levels in the samples it can be stated that the tablet tabletop sweetener stands out for its very high values. This can be justified by the fact that the liquid and powder form of this type of product are not used in a very controlled way and therefore they could be used in larger amounts by consumers. On the other hand, it is easier to dispense individual units of tablet sweeteners, which may be the main reason for the different levels observed. Although the levels of cyclamate in this sweetener have a logical justification, the presence of saccharine, as indicated on the label, makes this sample almost exclusively composed of high-intensity sweeteners.

\section{Exposure assessment}

Due to the lack of data showing the average intake of tabletop sweeteners in Portugal, it is not possible to evaluate the impact of these results on dietary exposure. However, if the average cyclamate concentration determined for each brand is taken into account, together with its ADI of $7 \mathrm{mg} \mathrm{kg}^{-1}$ and a weight range for an adult of $60-75 \mathrm{~kg}$, the minimum intake necessary to reach the ADI can be estimated and, thus, the risk that the intake of each tabletop sweetener brand may have on human health.

With this calculation it was stated that the consumption of the powder, liquid 1 and 2 tabletop sweetener brands could not easily reach the ADI for the weight range considered. On the other hand, regarding the very concentrated tablet brand, the number of units necessary to ingest would range approximately from 11 to 13 . Considering diabetic consumers who are most likely to use tabletop sweeteners daily, for instance in every hot liquid drink, it can be said that the concentration of cyclamate in this brand may put at risk compliance with the ADI. Furthermore, the intake of this tabletop sweetener by children would reach their ADIs even more easily due to their lower body weight. It should be noted that the intake of these hypothetical dose excludes the contribution of other possible sources of sodium cyclamate, which must be taken into account when estimating the intake of sweetener (e.g., soft drinks).

To date, no study has reported a cyclamate intake higher than the ADI for adults (Renwick 2006; VKM 
2014). However, studies with children showed for higher percentiles intakes of cyclamate exceeding its ADI (FSANZ 2004; Leth et al. 2007; Dewinter et al. 2016). It should be noted that these exposure studies do not take into account all types of foods containing cyclamate, such as tabletop sweeteners.

\section{Variability of cyclamate content}

By using the ANOVA statistical test, the variability of the concentration of cyclamate between batches and in the same batch of the four brands was assessed. Within the same batch there was homogeneity for the powder, tablet and liquid 2 tabletop samples, but not for the liquid 1 sample. Between different batches, only the powder and tablet samples showed homogeneity. The results of this assessment may indicate a lack of a strict control in the manufacturing process of the liquid tabletop brands. This scenario may be justified by the absence of maximum levels of use for cyclamate in this type of products. Furthermore, the fact that cyclamate has a low sweetening power means that the overall sweetness of the final product would not be significantly affected by small differences in the amount used in each batch.

\section{Conclusions}

The described method is adequate for the analysis of cyclamate in tabletop sweeteners and meets the requirements of validation and quality control proposed for these procedures.

From the sample analysis it was clear that there is a great variation of cyclamate content between the different tabletop sweeteners. This variation was observed not only between different matrices but also in the same food matrix (liquid 1 and 2 samples), which is a natural consequence of the lack of legislation concerning these type of products. This observation leads to the conclusion that the actual content of cyclamate in a given tabletop sweetener brand depends on a set of uncontrolled variables, such as the producers' requirements and the type of matrix in production. Thus, there is a real risk of surpassing the $\mathrm{ADI}$ of cyclamate.

To date, no studies have been published in Portugal that focus on either the determination of cyclamate content in tabletop sweeteners or their impact on dietary exposure. This reveals the importance of conducting studies to estimate the intake of this sweetener through the consumption of such products. It appears that there is a risk of exceeding the ADI for cyclamate by adults or children by consumption of tabletop sweeteners available in the market. In this way, it is important to conduct more studies to see if there is real need to change the legislation regarding the use of intense sweeteners in these sugar substitutes to avoid possible risks to human health.

Possible changes could consist in the establishment of MPLs for high-intense sweeteners in tabletop sweeteners to prevent the occurrence of excessively concentrated brands.

Moreover, the mandatory labelling of its exact content would, at least, enable consumers to make a more informed and controlled purchase and intake of this type of product.

\section{Disclosure statement}

No potential conflict of interest was reported by the authors.

\section{References}

Choi MMF, Hsu MY, Wong SL. 2000. Determination of cyclamate in low-calorie foods by high-performance liquid chromatography with indirect visible photometry. Analyst. 125:217-220.

De La Peña C. 2010. Diet men. The food-pharma origins of artificially sweetened products. In: De La Peña C, editor. Empty pleasures: the story of artificial sweeteners from saccharin to splenda. Chapel Hill (NC): The University of North Carolina Press; p. 65-104.

Dewinter L, Casteels K, Corthouts K, Van de Kerckhove K, Van der Vaerent K, Vanmeerbeeck K, Matthys C. 2016. Dietary intake of non-nutritive sweeteners in type 1 diabetes mellitus children. Food Addit Contam Part. 33:19-26.

EC. 1994. European Commission. European Parliament and Council Directive 94/35/EC of 30 June 1994 on sweeteners for use in foodstuffs. Off J Eur Union. L237:3-12.

EC. 1996. European Commission. Directive 96/83/EC of the European Parliament and of the Council of 19 December 1996 amending Directive 94/35/EC on sweeteners for use in foodstuffs. Off J Eur Union. L048:16-19.

EC. 2003. European Commission. Directive 2003/115/EC of the European Parliament and of the Council of 22 December 2003 amending Directive 94/35/EC on sweeteners for use in foodstuffs. Off J Eur Union. L24:65-71.

EC. 2008. European Commission. Regulation No 1333/2008 of the European Parliament and of the Council of 16 
December 2008 on food additives. Off J Eur Union. L354:16-33.

EC. 2011. European Commission. Commission Regulation (EU) No. 1129/2011 amending Annex II to Regulation (EC) No. 1333/2008 of the European Parliament and of the Council by establishing a Union list of food additives. Off J Eur Union. L295:1-177.

EN. 12857. 1999. Determination of cyclamate in foodstuffsby method by high performance liquid chromatography. Brussels: European Committee for Standardization.

EN. 1379. 1996. Determination of cyclamate and saccharin in liquid table top sweetener preparations - method by high performance liquid chromatography. Brussels: European Committee for Standardization.

EUFIC. 2012. European Food Information Council. Benefits and safety of low calorie sweeteners, EUFIC review. Available from: http://www.eufic.org/article/en/page/RARCHIVE/ expid/Benefits_Safety_Low_Calorie_Sweeteners/.

Food Standards Australia New Zealand (FSANZ). 2004. Consumption of intense sweeteners in Australia and New Zealand: benchmark Survey 2003. Evaluation Report Series No. 8. Melbourne: FSANZ.

Hauck M, Köbler H. 1990. Determination of cyclamate in complex matrix using HPLC after column derivatization with 4-fluoro-7-nitrobenzofurazan. Eur Food Res Technol. 191:322-324.

Herrmann A, Damawandi E, Wagmann M. 1983. Determination of cyclamate by high-performance liquid chromatography with indirect photometry. J Chromatogr. 280:85-90.

Huang Z, Ma J, Chen B, Zhang Y, Yao S. 2006. Determination of cyclamate in foods by high performance liquid chromatography-electrospray ionization mass spectrometry. J Anal Chim Acta. 555:233-237.

IPAC. 2007. Guia para a Quantificação de Incerteza em Ensaios Químicos. Caparica: Instituto Português de Acreditação.

ISO. 2012. International Sugar Organization. Alternative sweeteners in a high sugar price environment. Mark Eval Consum Stat Comm. 12:1-53.

Kishi A, Bizzari S, Janshekar H. 2010. High-intensity sweeteners. In: Chemical economics handbook. Available from: https://www.ihs.com/products/high-intensity-sweetenerschemical-economics-handbook.html.

Kokotou MG, Asimakopoulos AG, Thomaidis N. 2013. Sweeteners. In: Nollet LML, Toldrá F, editors. Food analysis by HPLC. 3rd ed. Boca Raton (FL): CRC Press; p. 493-514.
Leth T, Fabricius N, Fagt S. 2007. Estimated intake of intense sweeteners from non-alcoholic beverages in Denmark. Food Addit Contam. 24:227-235.

Renwick AG. 2006. The intake of intense sweeteners - an update review. Food Addit Contam. 23:327-338.

Sakai H, Yamashita A, Tamura M, Uyama A, Mochizuki N. 2015. Simultaneous determination of sweeteners in beverages by LC-MS/MS. Food Addit Contam Part. 32:808-816.

SCF. 2000. European Commission. Revised opinion on cyclamic acid and its sodium and calcium salts. Available from: https://ec.europa.eu/food/sites/food/files/safety/docs/scicom_scf_out53_en.pdf.

Shah R, Farris S, Jager LSD, Begley TH. 2015. A novel method for the simultaneous determination of 14 sweeteners of regulatory interest using UHPLC-MS/MS. Food Addit Contam Part. 32:141-151.

Shah R, Jager LSD, Begley TH. 2014. Development and single-laboratory validation of an improved method for the determination of cyclamate in foods using liquid chromatography/tandem mass spectrometry. J AOAC Int. 97:1651-1655.

Szmrecsányi T, Alvarez VMP. 1998. The search for a perfect substitute: technological and economic trajectories of synthetic sweeteners, from Saccharin to Aspartame (c.18801980). Available from: http://portal.ige.unicamp.br/pt-br/ biblioteca/acervo-cdpct/search-perfect-substitute-technolo gical-and-economic-trajectories-synthetic.

Vistuba JP, Dolzana MD, Vitali L, Oliveira MAL, Micke GA. 2015. Sub-minute method for simultaneous determination of aspartame, cyclamate, acesulfame- $\mathrm{K}$ and saccharin in food and pharmaceutical samples by capillary zone electrophoresis. J Chromatogr A. 1396:148-152.

VKM. 2014. Risk assessments of cyclamate, saccharin, neohesperidine DC, steviol glycosides and neotame from soft drinks, "saft" and nectar. Available from: http://www.vkm. no/dav/282e7c5098.pdf:Vitenskapskomiteen for mattryg ghet/the Norwegian scientific committee for food safety.

Wasik A, McCourt J, Buchgraber M. 2007. Simultaneous determination of nine intense sweeteners in foodstuffs by high performance liquid chromatography and evaporative light scattering detection - development and singlelaboratory validation. J Chromatogr A. 1157:187-196.

Zygler A, Wasik A, Kot-Was A, Ameśn J. 2011. Determination of nine high-intensity sweeteners in various foods by high-performance liquid chromatography with mass spectrometric detection. J Anal Bioanal Chem. 400:2159-2172. 